\title{
New product introduction and diffusion with costly search
}

\author{
Chung Yi Tse ${ }^{1}$ \\ University of Hong Kong, \\ 26 February, 2004
}

\begin{abstract}
Will the low search cost in the new economy help speed up new product introduction? The usual model of product market search suggests that a low search cost can turn out to have detrimental incentives on innovation and new product introduction as the low search cost erodes firms' market power, attenuating the profit from innovation. This usual model, however, misses the important dimension of product market search that how often it pays to search depends on the magnitude of the search cost. This paper studies a model of monopolistic competition with costly search, where the point of departure is that of a fixed cost of a shopping trip. With this fixed cost, the optimal search frequency is tied to the magnitude of the search cost. In this environment, a low search cost could turn out to be favorable to innovation. At a low search cost, consumers search more often, speeding up the diffusion of new products and possibly resulting in higher profits for firms, despite the erosion of market power.
\end{abstract}

JEL classifications: L10, L11, O31, D40

Key words: product market search, innovation, new product introduction.

\footnotetext{
${ }^{1}$ Correspondence: School of Economics and Finance, University of Hong Kong, Pokfulam Road, Hong Kong. email: tsechung@econ.hku.hk, tel: (+852) 2859-1035, fax: (+852) 2548-1152.
} 


\section{INTRODUCTION}

Will the low search cost in the new economy help speed up new product introduction? In the usual model of product market search with either homogeneous or differentiated products, as a result of a decline in the search cost of consumers, there will be a lower equilibrium price due to the erosion of market power. ${ }^{2}$ In this way, a low search cost could well slow down innovation in general and new product introduction in particular insofar as it attenuates the possible profits to be earned from innovation.

This line of reasoning is counterintuitive to many. And it only seems reasonable that a low search cost, in better facilitating the matching of goods and consumers, should provide greater incentives for investment in new product development. This paper suggests a plausible mechanism by which this can be the case.

The analysis is based on the conjecture that in a completely stagnant world, all search activities should eventually come to an end. Consumers may still have to physically visit (the lowest-priced) stores for making purchases. But these are not search activities as such, as there will be no elements of discovery in these visits. The need for search only arises when existing products become obsolete and new products get introduced over time. So far as search is costly, consumers shall only find it worthwhile to initiate search when they expect that there are sufficiently many new products introduced since the last time they searched. A more rapid pace of new product introduction then makes it optimal for consumers to search more often. Contrariwise, the profit from new product introduction depends on how fast the new product may diffuse to its potential customers. If a firm's customers search more often, there will be more rapid diffusion and greater profits. Where the frequency of

\footnotetext{
${ }^{2}$ For models with homogeneous products, see Reinganum (1979) and Fishman and Rob (1995), among others. For models with differentiated products, see Wolinsky (1984) and Anderson and Renault (1999).
} 
search on the part of consumers is tied to the pace of new product introduction, a low search cost can easily turn the environment favorable for new product introduction. As the search cost comes down, consumers search more often, which in turn will speed up the diffusion of new products, raising profits as a result.

This paper sets out to formalize these arguments in a model of monopolistic competition with costly search. The point of departure from the usual model of product market search is the assumption of a fixed cost of a shopping trip. When search exhibits increasing returns arising from the fixed cost of a shopping trip, sequential search, whereby consumers sample every time new offers arrive, may no longer be optimal. With a sufficiently large fixed cost, the optimal search policy is a quasifixed-sample-size search rule. Analogous to a $S-s$ type inventory policy, the optimal search rule is to wait out a given number of periods before sampling all brands introduced in the interim, where the optimal frequency of search depends on, among other factors, the pace at which new products are introduced and the fixed cost of a shopping trip. On the other hand, the profitability of new product introduction and hence, the pace at which new products are introduced go up when consumers search more frequently. In this environment, I demonstrate, via a numerical example, that a low search cost, despite eroding firms' market power, can raise the rate of new product introduction in equilibrium.

Notable among previous studies on how a lowering of search cost can raise investment in innovation is Pereira (2001), who explains that in the Reinganum (1979) model, where price dispersion arises out of differences in the marginal costs of production among firms, a lowering of search cost can result in an increase in cost reduction investment. In the Reinganum model, a lowering of search cost makes consumers choose a lower reservation price, eroding the profits of high cost firms, while leaving the profits of low cost firms unaffected. A falling search cost thus raises the possible increases in profits by becoming a low cost firm, giving rise to greater investment in 
cost reduction in equilibrium. ${ }^{3}$ In Tse (2003), I study, in an AK growth model, how a lowering of search cost may raise the growth rate of output through making the economy tend closer to the ideal of perfect competition.

The Pereira (2001) and the Tse (2003) models are variants of what may be termed the competitive pressure hypothesis, under which a low search cost, despite lowering the average returns, raises the marginal returns to investment. In Pereira (2001), there is greater urgency to invest in a low search cost environment, as the low search cost disproportionately eats into the profits of high cost firms. In Tse (2003), when facing more elastic demand curves, the marginal revenues of firms fall less rapidly with output, giving rise to greater investment incentives. Likewise, the marginal returns to investment in the present model can go up in a low search cost environment. The distinctive feature of this model is that, when the search efforts of consumers are made endogenous, the average returns to investment need not fall in the mean time. True, firms may only have lower market power. But there can be greater sales when the new products diffuse more rapidly through the more frequent search of consumers. For lack of a better name, I call this the market turnover hypothesis. While the competitive pressure hypothesis implies that search cost, markup, the stock market value of firms on the one hand, and innovation on the other hand should be negatively correlated, under the market turnover hypothesis, the correlation between the stock market value of firms and innovation induced by a falling search cost can be positive. This difference can then be the basis to distinguish the two hypotheses empirically.

The main analysis follows in the next section. Section 3 discusses the effects of a low search cost on market structure, profits, and innovation. Section 4 discusses the social optimum and Section 5 concludes with some brief remarks. All proofs not obvious from the discussion are relegated to the appendix.

\footnotetext{
${ }^{3}$ This idea has its historical origin in the replacement effect in the early $R \& D$ literature first explained in Arrow (1962).
} 


\section{MODEL}

\section{A. Basics}

Time is discrete and the horizon is infinite. The market is populated by a unit measure of risk-neutral consumers, each of whom has a unit demand for a differentiated good at any one time. The match between a given consumer and a given brand of the good, denoted as $\theta$, is an iid draw from a stationary distribution $F(\theta)$, where $F(0)=0$ and $F(B)=1$ for some $B<\infty$. Having purchased a given brand, it takes the consumer $K>1$ periods of time to consume the brand, during which she is completely satiated with any other brands of the good. Furthermore, once having consumed a brand, the consumer derives no additional utility from its repeated purchase. Specifically, a consumer derives the net surplus

$$
u=\theta-p
$$

at the moment a brand having a match value of $\theta$ and charged a price $p$ is purchased. Thereafter, the consumer stays dormant for $K$ periods before reentering the market.

When then consumer returns from having stayed dormant for the $K$ periods, she may pay some $s \geq 0$ to initiate search for a new unit in any period of time to follow. And once the consumer sinks the cost of initiating search for the period, she may sample all brands available for sale at the time at no additional cost. If no brands are deemed acceptable in the shopping trip, the consumer remains in the market to wait for brands that better match her taste to be introduced. In all, an active consumer faces two decisions in each period. The first is whether or not to initiate search at a cost of $s$. Conditional on having done so, the consumer would then decide on whether or not to buy the brand that best matches her taste. The decisions are 
made to maximize the expected discounted utility of consumption:

$$
U=E\left[-s \sum_{i=0}^{\infty} \beta^{\tau_{i}}+\sum_{j=0}^{\infty} \beta^{\tau_{j}}\left(\theta_{j}-p\right)\right],
$$

where $E$ is the expectation operator, $\beta<1$ the discount factor, $\tau_{i}$ the dates the consumer goes shopping, $\tau_{j}$ the dates when purchases of the good are made, and $\theta_{j}$ the match of the $j$ th purchase.

The next section demonstrates that in an environment under which some $e$ new brands are introduced in each period and sold at the same price $p$ over time, the optimal search policy is such that the consumer initiates search once every $T$ periods and accepts all matches at which $\theta$ exceeds some reservation match $\bar{\theta}$. Optimal search is thus a pair $\{\bar{\theta}, T\}$ in response to a given $\{p, e\}$ pair. In turn, this implies a certain fraction, $N(T, \bar{\theta})$, of all consumers will choose initiating search in each period.

While consumers take as given the $\{p, e\}$ pair, these are choice variables for firms. In particular, I assume monopolistic competition among firms in which each firm can introduce a new brand at a cost equal to some $c>0$ and produces it at 0 marginal cost. Taking as given the number of searching consumers in each period of time and the optimal search behavior of each as governed by the pair $\{\bar{\theta}, T\}$, and the price charged by other firms, each firm chooses the price to charge for its brand to maximize discounted profit. In equilibrium, the rate of entry is pinned down by a zero profit condition.

As will become clear in the following, firms have incentives to drop the prices of their brands over time as the brands age, a situation reminiscent of the pricing problem facing a durable good monopolist. If firms do drop the prices of their brands over time, the consumer search problem is not time-stationary. To shut down all sources of non-stationarity, first I shall assume that when a consumer first becomes active after having stayed dormant for $K$ periods - the time it takes for her to consume the last unit purchased, she has no interests at all in any and all brands introduced in the 
interim. Second, firms are assumed to be able to commit to the first best but possibly not time-consistent pricing policy. These assumptions are admittedly ad hoc, but the analysis is not tractable without them. And they do not seem to be critical for the major conclusions to be derived.

In all, equilibrium in the monopolistic competition under these assumptions can be characterized by a pair of price and rate of entry, $\{p, e\}$, in response to a given $\{\bar{\theta}, T\}$ pair. The full equilibrium then is defined as a quadruple $\{\bar{\theta}, T, p, e\}$ that simultaneously satisfies optimal consumer search and equilibrium in the monopolistic competition.

Under the assumptions on preference and technology, new brands are not any better than old ones. They are just different. The analysis, strictly speaking, applies only to markets where new varieties are introduced solely for their novelty values. These may be the markets for DVD, fashion, or pulp friction. Perhaps a more useful analysis would be a model in which new brands stochastically improve upon old ones. In such a model, consumers would choose to upgrade to better brands from time to time, and the incentives to search for better brands to replace the old ones could depend on how costly it would be to search for these better brands. This analysis turns out to be much more difficult to handle, however, as it necessarily involves solving a nonstationary consumer search problem. As a first stab to understand the relationship between costly search on the part of consumers and the incentives to introduce new products on the part of firms with endogenous search efforts, the present setting provides a much more tractable framework.

\section{B. The consumer's problem}

In this section, I begin analyzing the consumer search problem when consumers face an environment in which $e$ new brands, each of which sells at the same $p$ over time are introduced in each period. In this case, if a consumer found a given brand not 
acceptable in a previous shopping trip, the same brand shall remain not acceptable in the upcoming shopping trip if they do sell at the same price over time. Hence, the number of brands that the consumer may choose from in the shopping trip is effectively restricted to the number of brands introduced since she last searched. If the consumer last searched some $t \geq 1$ periods ago, the choices will be among the $e t$ brands introduced in the interim.

Denote $U(t)$ as the expected discounted utility of consumption of an active consumer at the beginning of a period in which she last searched $t$ periods ago. If she chooses to initiate search in the given period, there is first the trip cost to be incurred, followed by the expected payoff of search, denoted as $W(t)$. The decision of whether or not to initiate search is governed by

$$
U(t)=\max \{\beta U(t+1), W(t)-s\}
$$

Suppose the consumer has chosen going shopping, and the best match among the et brands happens to equal to some $\theta$. If $\theta$ is deemed not to be an acceptable match, the consumer remains in the market, the value of which is given by $U(1)$ - the discounted utility of consumption where the last shopping date was the previous period. If she makes the purchase, the consumer collects the payoff $\theta-p$, stays dormant for $K$ periods, and then reenters the market. As stated in the previous section, I shall assume that, upon reentering the market, the consumer has no interest in any of the brands introduced in the interim. Under this assumption, the value of reentering the market after having stayed dormant for $K$ periods is the same as the value of having just made an unsuccessful shopping trip in the previous period given by $U(1){ }^{4}$ Thus,

\footnotetext{
${ }^{4}$ Without this assumption, a firm would be selling to two types of buyers in each period. The first type - consumers who reenter the market after having stayed dormant for $K$ periods, will be choosing among all brands introduced since they withdrew from the market $K$ periods ago. The second type is made up of consumers who did not buy the last time they searched, say $T$ periods ago.
} 
the decision of whether or not to make the purchase is governed by

$$
w(\theta)=\max \left\{\theta-p+\beta^{K} U(1), \beta U(1)\right\}
$$

I may then define the expected payoff of going shopping as

$$
W(t)=\int w(\theta) d F(\theta)^{e t}
$$

Clearly, the optimal policy of (3) is a reservation match policy, under which the consumer should make the purchase if and only if $\theta \geq \bar{\theta}$, where $\bar{\theta}$ satisfies $^{5}$

$$
\bar{\theta}-p=\left(\beta-\beta^{K}\right) U(1)
$$

The reservation match, $\bar{\theta}$, depends on the value of continuing search, $U(1)$, which is given by (2) with $t=1$. To characterize $\bar{\theta}$, it is first necessary to characterize the optimal policy of (2). To this end, it is useful to establish the following:

Depending on whether $T_{<}^{>} K$, the second type of consumers could be choosing among a larger or a smaller number of brands in the period concerned. In either case, the profit-maximzing price for the two groups must differ, with a possibly lower price for the group where each member faces more choices. A firm's demand in the given period is the sum of the demands of the two groups. Over time as a brand ages, the first group could make up an increasingly larger fraction of its demand. Then even the first-best, possibly not time-consistent, pricing policy is such that the price of a brand would fall over time.

${ }^{5}$ This underscores the role played by the assumption that the consumption of a brand takes $K>1$ periods of time. Setting $K=1$, (5) shows that the consumer will make the purchase so long as $\theta \geq p$. With the reservation match independent of $U(1)$ and therefore $s$, the equilibrium price will not respond to changes in $s$. A lower search cost as such, shall not, affect market power one way or another, a manifestation of Diamond's (1971) famous result that market power stays at the maximum level so long as search cost is positive. Intuitively, when the consumer shall retain the same unit for longer than a single period, there is an opportunity cost of not able to consume a better match in the upcoming $K$ periods when making the purchase in the current period. This makes the consumer holds out for a better match, and this option is of greater value as $s$ declines, giving rise to a link between search cost and the extent of market power. 


\section{Lemma 1}

$$
W(t)=\int_{\bar{\theta}}\left(1-F(\theta)^{e t}\right) d \theta+\bar{\theta}-p+\beta^{K} U(1)
$$

\section{Lemma 2}

(a) If $W(t)-s \geq \beta U(t+1)$, then $W(t+1)-s \geq \beta U(t+2)$. That is, if the consumer finds it optimal to initiate search at some $t$, she finds it optimal to do so at all $t^{\prime}>t$.

(b) The optimal policy of the search problem defined in (2) is a $S-s$ type policy, under which the consumer finds it optimal to initiate search once every $T$ periods, for some $T \geq 1$.

Having established the optimality of a $S-s$ type policy for the search problem in (2), I can proceed to characterize $\bar{\theta}$. First, $U(1)$ in (3) and (5) becomes $\beta^{T-1} U(T)$, whereas $U(T)=W(T)-s$. Substituting lemma 1 for $W(T)$ and simplifying, the indifference condition in (5) becomes

$$
\frac{\bar{\theta}-p}{1-\beta^{K-1}}=\frac{\beta^{T}}{1-\beta^{T}}\left(\int_{\bar{\theta}}\left(1-F(\theta)^{e T}\right) d \theta-s\right) .
$$

This condition defines the reservation match, $\bar{\theta}$, for a given $T$. By lemma $2, T$ is the smallest integer that satisfies

$$
W(T)-s \geq \beta(W(T+1)-s) .
$$

Substituting lemma 1 for $W(T)$ and $W(T+1)$ respectively and simplifying using (6) yields

$$
\frac{\beta^{T}}{1-\beta^{T}}\left(\int_{\bar{\theta}}\left(1-F(\theta)^{e T}\right) d \theta-s\right) \geq \frac{\beta^{T+1}}{1-\beta^{T+1}}\left(\int_{\bar{\theta}}\left(1-F(\theta)^{e(T+1)}\right) d \theta-s\right) .
$$

This condition is difficult to work with because of the integer constraint on $T$. To simplify, I shall derive an analogous condition that allows $T$ to take on non-integer 
values for the following. To this end, notice that (7) says that $T$ should be chosen to maximize the right side of (6), where $\bar{\theta}$ is implicitly defined as the solution to (6) itself. Hence, I may differentiate the right side of (6) with respect to $T$ to obtain the condition:

$$
\left(\int_{\bar{\theta}}\left(1-F(\theta)^{e T}\right) d \theta-s\right) \ln \beta-\left(1-\beta^{T}\right) e \int_{\bar{\theta}} F(\theta)^{e T} \ln F(\theta) d \theta=0
$$

whose left side is decreasing in $T$ throughout for each $\bar{\theta}$ lying in the interior of the support of $F(\theta)$. This guarantees that (8), in combination with (6), is also sufficient for an interior optimum.

Figure 1 depicts the two sides of (6) as a function of $\bar{\theta}$ for some given $T$, where the point of intersection defines the optimal reservation match for the assumed $T$. And then according to $(7)$ or its equivalence that allows for non-integer valued $T,(8)$, $T$ should be chosen so as to make the curved-line that is the right side of (6) rise highest. In this way, $\frac{\bar{\theta}-p}{1-\beta^{K-1}}$, which is the value of search at the outset, is maximized. ${ }^{6}$

${ }^{6}$ Where the distribution of offers is continuous, the value of search at the outset, as defined in (1), is equal to the stopping value at the optimum, a well-known result first established in Lippman and McCall (1974). In the present case, given the optimality of a $S-s$ type policy, (3) can be rewritten as

$$
w(\theta)=\max \left\{\theta-p+\beta^{K+T}\left(\int w\left(\theta^{\prime}\right) d F\left(\theta^{\prime}\right)^{e T}-s\right), \beta^{T}\left(\int w\left(\theta^{\prime}\right) d F\left(\theta^{\prime}\right)^{e T}-s\right)\right\}
$$

with an indifference condition: $\bar{\theta}-p=\beta^{T}\left(1-\beta^{K}\right)\left(\int w\left(\theta^{\prime}\right) d F\left(\theta^{\prime}\right)^{e T}-s\right)$. The value of search at the outset (before the first search takes place) is $\bar{\theta}-p+\beta^{K+T}\left(\int w\left(\theta^{\prime}\right) d F\left(\theta^{\prime}\right)^{e T}-s\right)$. Substituting in the indifference condition yields $\frac{\bar{\theta}-p}{1-\beta^{K-1}}$. 


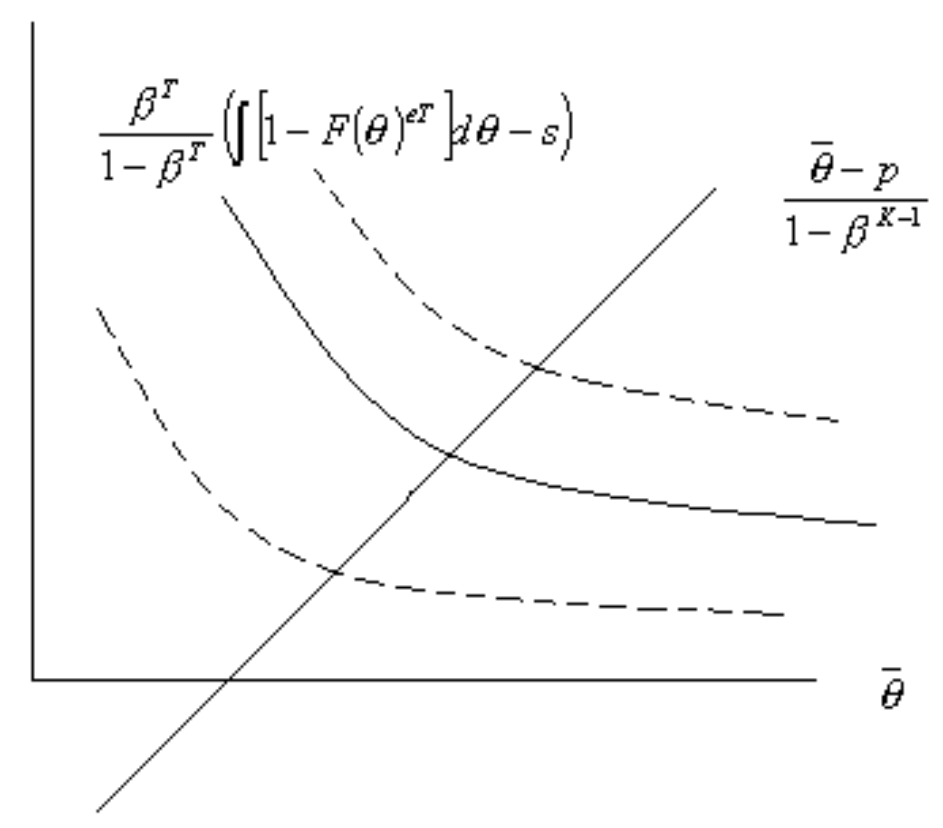

Figure 1: Optimal search

Finally, I should continue to respect the constraint that $T \geq 1$, for a value of $T$ below unitary is non-sensical. Thus, I shall take $T=1$ as the optimal search frequency if the left side of (8) should fall below 0 at $T=1$. With (6) and (8) two equations in $\bar{\theta}$ and $T$ characterizing the consumer's optimum:

\section{Proposition 1}

(a) $T=1$ for sufficiently small $s$.

(b) Conversely, for a sufficiently large $s$, optimal search is given by a $T>1$. In this case: (i) increases in $s$ raise $T$ but lower $\bar{\theta}$, (ii) increases in $p$ raise $T$ and $\bar{\theta}$, and (iii) increases in $e$ lower $T$ but raise $\bar{\theta}$.

With $s=0$, that $T=1$, as established in proposition $1(\mathrm{a})$, is simply a manifestation of the optimality of sequential search when search exhibits no scale economies. In this case, the optimal search strategy is to decide on whether to continue searching 
every time new offers are available for consideration. ${ }^{7}$

When the non-convexity introduced by a $s>0$ is sufficiently pronounced, sequential search becomes no longer optimal. In this case, the right side of (6) and so the curved-line in figure 1 may well rise highest at some $T>1$. Indeed, as established in proposition 1(b), as $s$ increases, the consumer would find it optimal to wait out an increasingly long interval between two shopping dates, so as to draw a larger sample in one search. Even though there is now a larger sample to choose from in each search, the reservation match $\bar{\theta}$ falls in the meantime. In terms of figure 1 , as $s$ rises, the right side of (6) and hence the curved-line shifts down at the initial $T$. The optimal upward adjustment in $T$ would raise the curved-line somewhat but the increase cannot possibly compensate for the decline due to a larger $s$. As a result, there will be a smaller $\bar{\theta}$, commensurate with the fact that the value of search declines in the now less favorable environment.

An increase in $p$, in raising $T$, similarly lowers the optimal shopping frequency. In this case, $\bar{\theta}$ rises in response. This increase is not, however, an increase in the value of search as the whole of $\bar{\theta}-p$ should have fallen. Intuitively, the higher price makes going shopping less attractive. In response, the consumer finds it optimal to search less frequently. On the other hand, as the net surplus the consumer may derive from purchasing any given brand falls at the higher price, she would find making the purchase optimal only at a better match.

Finally, the consumer will find it optimal to search more frequently as $e$ increases, as a more rapid pace of new product introduction raises the cost of delaying sampling. In terms of figure 1 , the increase in $e$, coupled with the optimal downward adjustment in $T$, raises the right side of (6) and therefore the curved-line in figure 1, yielding a larger $\bar{\theta}$ as a result. In the now more favorable environment where the consumer faces a better distribution of offers, the value of search should only go up.

\footnotetext{
${ }^{7}$ Kohn and Shavell (1974).
} 


\section{Turnover of consumers}

Let $n_{\tau}(t), 1 \leq t \leq T$, be the beginning of period $\tau$ number of consumers who last searched $t$ periods ago but failed to find an acceptable match then. For $1<t \leq T$, the evolution of $n_{\tau}$ follows

$$
n_{\tau}(t)=n_{\tau-1}(t-1)
$$

At the beginning of period $\tau$, all consumers who have stayed put for $T$ periods find it optimal to initiate search. The number of consumers searching in the period is thus

$$
N_{\tau}=n_{\tau}(T)
$$

Among all consumers who searched last period, $N_{\tau-1}$, a fraction $F(\bar{\theta})^{e T}$ failed to find an acceptable match and remained in the market. The successful shoppers $K$ periods earlier, numbering $N_{\tau-K}\left(1-F(\bar{\theta})^{e T}\right)$, will reenter the market in period $\tau$ to search for a replacement for their last unit. Since each is assumed not to be interested in any of the currently available brands, these consumers would behave as if they were unsuccessful shoppers in $\tau-1$. With a slight abuse of notation, I may define

$$
n_{\tau}(1)=N_{\tau-1} F(\bar{\theta})^{e T}+N_{\tau-K}\left(1-F(\bar{\theta})^{e T}\right) .
$$

Under the normalization that the population of consumers is unitary:

$$
\sum_{t=1}^{T} n_{\tau}(t)+\sum_{t=1}^{K-1} N_{\tau-t}\left(1-F(\bar{\theta})^{e T}\right)=1 .
$$

In the steady state when $n_{\tau}(t)=n_{\tau-1}(t),(9)-(12)$ imply that the number of consumers searching in each period is given by

$$
N=\frac{1}{T+(K-1)\left(1-F(\bar{\theta})^{e T}\right)} .
$$

Individual behavior affects aggregate outcome as to the number of consumers searching in each period in two distinct manners. First, when each active consumer 
chooses more frequent search (a smaller $T$ ), there tends to be more searching consumers in each period. Second when matches are accepted with a lower probability (a smaller $\left.1-F(\bar{\theta})^{e T}\right)$, at each moment in time, there will be fewer consumers staying dormant, and other things equal, more will choose searching in each period. In all, $N$ is decreasing in $e$ and $T$ but increasing in $\bar{\theta}$.

\section{Monopolistic competition}

In the monopolistic competition, each firm takes as given the number of competing brands offered for sale in each period, the price charged by other firms, $p$, the search frequency, $T$, and reservation match of consumers, $\bar{\theta}$, and the number of searching consumers in each period, $N$, as determined by (13), which is a function of $T$ and $\bar{\theta}$. Then it chooses the price to charge for its brand in each period to maximize discounted profit.

If prices are indeed stationary over time, the searching consumers in a given period will only be interested in brands introduced since they last searched $T$ periods ago. Brands introduced earlier can find no potential buyers. This necessitates the shelf-life of a brand be restricted to $T$ periods, after which the brand is effectively obsolete. If there are $e$ brands introduced in each period and each has a shelf-life of $T$ periods, at any one time, the number of brands competing for the business of the searching consumers in the period is down to $e T$. In each period of a brand's shelf-life then, it will be competing with $e T-1$ other brands for the business of each searching consumer in the period. ${ }^{8}$ In particular, a given brand $i$, when it is charged a price $p_{i}$, will sell to

\footnotetext{
${ }^{8}$ Take $T=2$ for example. In period $\tau$, the shoppers are consumers who last searched in period $\tau-2$, each of whom in the upcoming shopping trip will only be interested in brands of vintages $\tau-1$ and $\tau$. Brands of vintages $\tau-2$ and earlier will find no potential buyers, while each active brand, numbering $2 e$ in total, is competing with each of the other $2 e-1$ brands for the business of each shopper. In period $\tau+1$, the shoppers will be consumers who last searched in $\tau-1$, each of
} 
consumers for whom it offers the highest net surplus, i.e., $\theta_{i}-p_{i}>\max _{j \neq i}\left\{\theta_{j}-p\right\}$, in addition to satisfying the consumers' reservation match policy: $\theta_{i}-p_{i} \geq \bar{\theta}-p$. With $N$ given by (13), this implies that in each period of brand $i$ 's shelf-life, its demand at price $p_{i}$ is stationary at the level:

$$
D_{i}\left(p_{i}, p, \bar{\theta}, e, T\right)=\frac{\int_{\bar{\theta}-p+p_{i}} F\left(\theta-p_{i}+p\right)^{e T-1} d F(\theta)}{T+(K-1)\left(1-F(\bar{\theta})^{e T}\right)} .
$$

With a fixed cost of new product introduction equal to $c$ and a 0 marginal cost of production, the discounted profit of the brand is given by

$$
\Pi_{i}(p, \bar{\theta}, T, e)=\max _{p_{i}}\left\{\frac{1-\beta^{T}}{1-\beta} \frac{p_{i} \int_{\bar{\theta}-p+p_{i}} F\left(\theta-p_{i}+p\right)^{e T-1} d F(\theta)}{T+(K-1)\left(1-F(\bar{\theta})^{e T}\right)}\right\}-c
$$

which will similarly yield a time-stationary price. It should be pointed out that the pricing policy underlying (14) is not time-consistent. A consumer sampling a brand the second time around will never find it an acceptable match at the same price she was first quoted if she did not find it an acceptable match in the first place. It is this presumption that limits a brand's shelf-life to $T$ periods, which can possibly be relaxed if the firm lowers the brand's price over time as it ages. But following such a time-varying pricing policy will adversely affect the brand's initial demand. As first explained in Bulow (1984) in the context of a durable good monopolist, firms are better off resisting the temptation to drop prices over time so as to avoid competing with itself over the same pool of customers in between periods. Essentially, underlying (14) is the assumption that firms can commit to the time-inconsistent but optimal pricing policy.

$\overline{\text { whom will only be interested in vintages }} \tau$ and $\tau+1$ brands. Likewise, each active brand will be competing with the other $2 e-1$ brands for the business of each shopper. In all, a vintage $\tau$ brand will have a shelf-life of 2 periods. And in each period of these 2 periods, it faces the same demand insofar as it may sell to the same number of potential buyers, each of whom may choose among the same number of competing brands. 
Taking first order condition of (14) and then setting the profit-maximizing price, $p_{i}$ to $p$ yield the equilibrium price in terms of $e, T$, and $\bar{\theta}$ :

$$
p=\frac{1-F(\bar{\theta})^{e T}}{e T\left(f(\bar{\theta}) F(\bar{\theta})^{e T-1}+\int_{\bar{\theta}} f(\theta) d F(\theta)^{e T-1}\right)},
$$

where $f(\theta)$ denotes the density function of $F(\theta) .{ }^{9}$ When consumers choose a higher reservation match that arises from a lower search cost, for example, a priori, one would expect a lower equilibrium price. Similarly, when consumers may choose among a greater number of brands in each shopping trip, there should be a lower equilibrium price too as a result of the more intense competition. The following lemma presents regularity conditions on $F(\theta)$ that guarantee the desired results.

Lemma 3 For all $e T \geq 2$ and all $\bar{\theta}$ in the support of $F($ ),

(a) a sufficient condition for $p$ to be decreasing in $\bar{\theta}$ is

$$
f(\bar{\theta})+\bar{\theta} f^{\prime}(\bar{\theta}) \geq 0
$$

(b) a sufficient condition for $p$ to be decreasing in $e$ and $T$ is

$$
\int_{\bar{\theta}} f(\theta)^{2} F(\theta)^{x}(1+(x+1) \ln F(\theta)) d \theta \geq 0
$$

for all $x \geq 0$.

In both cases, the conditions, which are satisfied by the uniform distribution, among possibly many others, require that the density not be falling too rapidly with $\theta$. To avoid dealing with a proliferation of possibilities, I shall assume that from this point onward, the conditions of the lemma hold. In this case, it can be verified that:

\footnotetext{
${ }^{9}$ With $F(\theta)$ continuously differentiable over the positive support of the distribution, the second order condition sufficient for a local optimum is guaranteed to be satisfied. See the proof of lemma 3.
} 
Lemma 4 In equilibrium, the discounted profit of a given brand is likewise decreasing in $e, T$, and $\bar{\theta}$.

With free entry in new product introduction, we must have the discounted profit of each brand non-positive in equilibrium. Setting $p_{i}=p$ in (14), the free entry condition that applies in an interior equilibrium can be written as:

$$
\frac{1-\beta^{T}}{1-\beta} \frac{p\left(1-F(\bar{\theta})^{e T}\right)}{e T\left(T+(K-1)\left(1-F(\bar{\theta})^{e T}\right)\right)}=c
$$

which, together with (15), pins down the rate of entry, e, given $T$ and $\bar{\theta}$ from consumers' optimal search. It follows from lemma 4 that:

Proposition 2 In the monopolistically competitive equilibrium, the rate of new product introduction, $e$, speeds up when consumers search more frequently (a smaller $T$ ) but will slow down when they set a higher standard, $\bar{\theta}$, for accepting a match.

\section{SEARCH COST, MARKET STRUCTURE, AND NEW PRODUCT INTRODUCTION}

The full equilibrium is defined as the quadruple $\{\bar{\theta}, T, e, p\}$ that simultaneously satisfies consumers' optimum as characterized by (6) and (8) and the monopolistically competitive equilibrium as characterized by (15) and (16) .

It is instructive to first examine the properties of an equilibrium where the search efforts of consumers are exogenously given, or equivalently that the product market search is restricted to be strictly sequential. Fixing $T=1$ and given $p$ and $e$, in this case, consumers' optimum is solely characterized by a $\bar{\theta}$ that solves (6), and the equilibrium simplifies to a triple $\{\bar{\theta}, p, e\}$ that simultaneously satisfies this condition and 
equilibrium in the monopolistic competition as characterized by (15) and (16). Further simplification can be obtained by substituting (15) into (6) and (16) respectively to yield a system of two equations:

$$
\begin{gathered}
\frac{1-\beta^{T}}{1-\beta} \frac{p(\bar{\theta}, e, T)\left(1-F(\bar{\theta})^{e T}\right)}{e T\left(T+(K-1)\left(1-F(\bar{\theta})^{e T}\right)\right)}=c, \\
\frac{\bar{\theta}-p(\bar{\theta}, e, T)}{1-\beta^{K-1}}=\frac{\beta^{T}}{1-\beta^{T}}\left(\int_{\bar{\theta}}\left(1-F(\theta)^{e T}\right) d \theta-s\right),
\end{gathered}
$$

in $\bar{\theta}$ and $e$, where $p(\bar{\theta}, e, T)$ denotes the right side of $(15)$ and where $T$ is fixed at 1 in this instance.

By virtue of proposition 2, (17) defines a negatively-sloped relationship between $\bar{\theta}$ and $e$. Call this the FF schedule. Intuitively, a higher $\bar{\theta}$ erodes firms' profits, and the zero profit condition that is (17) is only restored at a lower rate of entry.

Fixing $p$ at some arbitrary value, from proposition 1, the consumer's optimal response to an increase in $e$, as governed by (18), is to raise $\bar{\theta}$ and lower $T$. It is clear from figure 1 that $\bar{\theta}$ shall go up is independent of whether we allow $T$ to be endogenous or whether it is fixed at some arbitrary value as is assumed to be the case in this analysis. The real complication is that we are now taking into account the fact that an increase in $e$ will also lower $p$, and this tends to make consumers choose a smaller $\bar{\theta}$. It can be seen from (18) that this indirect effect will be negligible for a sufficiently small $\frac{1-\beta^{T}}{\left(1-\beta^{K-1}\right) \beta^{T}} .{ }^{10}$ Assuming this to be the case, (18) defines a positively-sloped relationship between $\bar{\theta}$ and $e$. Call this the $C C$ schedule. Intuitively, in response to a more rapid rate of new product introduction, consumers respond by raising the reservation match.

\footnotetext{
${ }^{10}$ Fixing $T=1$, this expression is small for large $K$ and for $\beta$ close to 1 .
} 


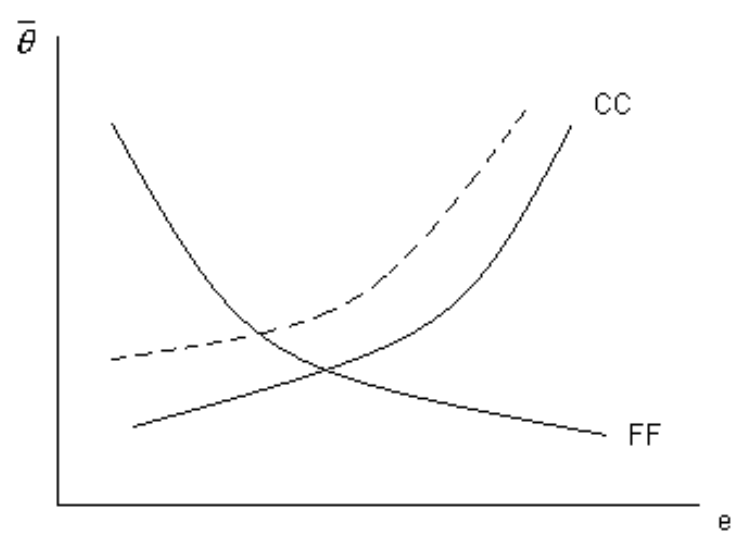

Figure 2: Equilibrium with fixed $T$

A non-trivial equilibrium, given existence, must then be unique, as shown in figure 2. Now consider a decline in $s$. The $F F$ schedule, as governed by (17), is not affected by any such changes, whereas the $C C$ schedule, as governed by (18), will shift up as shown in figure $2 .{ }^{11}$ Hence, we can conclude that

Proposition 3 Fixing the frequency of shopping at $T=1$, a decline in search cost, $s$, will result in consumers choosing a higher reservation match. In equilibrium, there will be a slower pace of new product introduction.

This result is what is expected of the usual model of product market search that assumes an environment in which optimal search is strictly sequential. In this case, at a lower search cost, consumers find it optimal to tighten up the reservation match,

\footnotetext{
${ }^{11}$ To see this, note that the decline in $s$ raises the right side of (18) for all $e$. If $\bar{\theta}$ were to decline at each $e$, the right side would go up even further, whereas the decline in $\bar{\theta}$ would serve to the lower the left side of the equation in the meantime.
} 
which serves to police firms' pricing and to lower the expected profit a given brand may earn from each searching consumer. With each and every active consumer assumed to choose searching in each period of time when $T$ is fixed at 1 , the expected discounted profit of a given brand goes down, resulting in a slower pace of new product introduction in equilibrium.

This model misses the important dimension of product market search that consumers may also optimize along the frequency of search. Allowing $T$ to be endogenously determined, the effect of a low search cost on new product introduction can be completely turned around. But first the uniqueness of equilibrium can no longer be so easily assured. With $T$ endogenous, the equilibrium is now made up of (8), which governs the consumer's optimal choice of $T$ in response to a given $e$, in addition to $(17)$ and $(18)$ in $\{T, \bar{\theta}, e\}$. Without examining the properties of this system in details, it is immediately possible to ascertain that a trivial equilibrium where $e=0$ and $T \rightarrow \infty$ always exists. With $e=0$, it never pays to search where search is costly. With $T \rightarrow \infty$ and so $N=0$, there will be no new product introduced in each period where new product introduction is costly, justifying an $e=0$.

This reasoning suggests that the multiplicity of non-trivial equilibria is a distinct possibility. To see this, say we start with some non-trivial equilibrium triple $\{T, \bar{\theta}, e\}$. Now in the consumers' optimum, $T$ is decreasing in $e$, and in the monopolistically competitive equilibrium, $e$ is likewise decreasing in $T$. There may then be an alternative equilibrium with a more rapid pace of new production introduction and more frequent search on the part of consumers. The possibility of multiplicity arises from the fact that, with the fixed cost of search, consumers' search and firms' new product introduction are strategic complements, and when the complementarity is sufficiently pronounced, a higher level equilibrium could emerge.

The possibility of multiple equilibria in a search and matching model is first raised in Diamond (1982) who assumes an increasing returns matching function. On a 
deeper level, the environment assumed in this paper resembles an increasing returns matching function. The non-convexity in consumers' search that is due to the fixed cost of a shopping trip turns an otherwise constant return environment into one where consumers' search exhibits increasing returns. The contribution of the present analysis is that it suggests a plausible manner in which the non-convexity in search and matching can arise - the fixed cost of initiating search.

Now suppose equilibrium is unique, so that comparative statics can be well-defined. How may a low search cost affect market structure and new product introduction in this environment? As $s$ falls, consumers not only will choose to raise the reservation match, but also will search more frequently. In turn, the more frequent search speeds up the diffusion of new products. True, the low search cost may erode firms' market power and tends to lower a brand's discounted profit. But the positive effect on a brand's profit arising from more frequent search may well more than compensate for the erosion of market power. The net effect is a possible increase in a brand's discounted profit at the existing rate of entry and a more rapid pace of new product introduction in equilibrium.

Figure 3 illustrates this possibility. The example assumes a uniform distribution of match on the unit interval, i.e., $F(\theta)=\theta$ for $\theta \in[0,1], \beta=0.999, K=10, c=0.001$, and $s=0.6 .{ }^{12}$ To analyze equilibrium in the $e-T$ space, first define the solution of $\bar{\theta}$ to (18) as $\bar{\theta}=\bar{\theta}(e, T)$, and then substitute it into (8) and (17) respectively. This results in a system of two equations in $e$ and $T$. Roughly speaking, the first equation, say the $C S$ schedule, characterizes the consumers' optimum, whereas the second equation, say the $F E$ schedule, characterizes equilibrium in the monopolistic competition.

\footnotetext{
${ }^{12}$ At $\beta=0.999$ and if the annual interest rate is $5 \%$, a period is about a week long.
} 


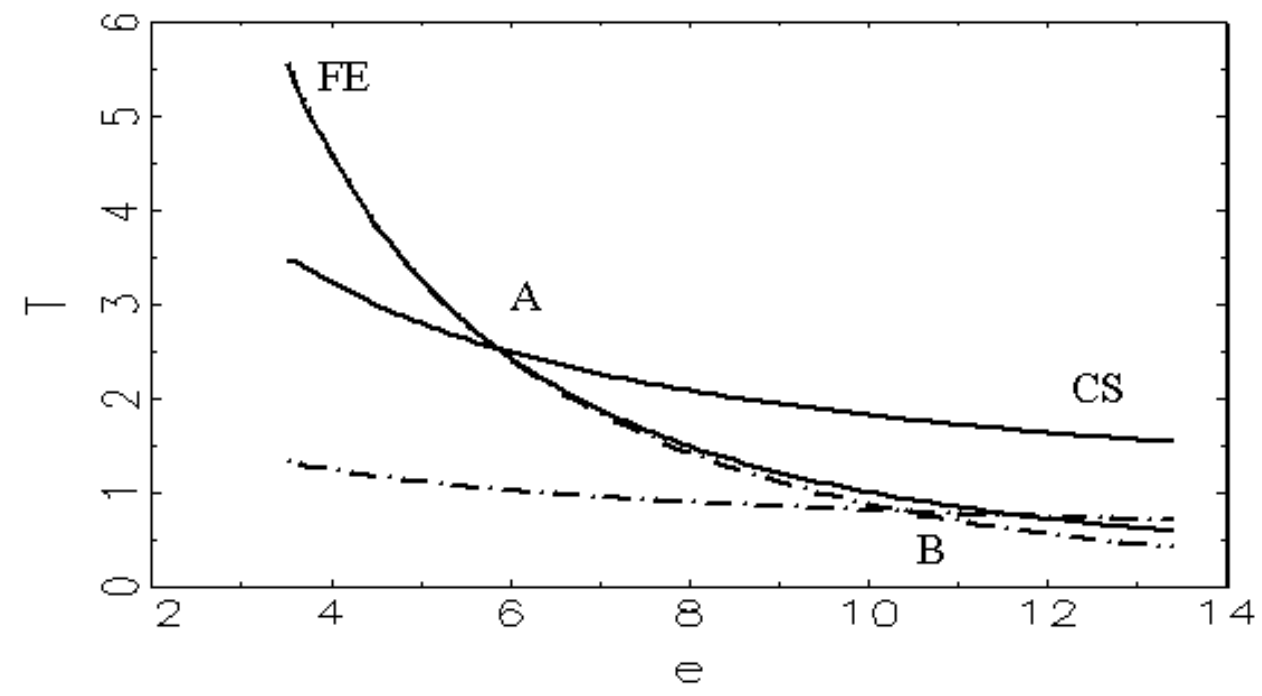

Figure 3: Full market equilibrium

The two schedules, FE and $C S$ are plotted as the two solid lines in Figure 3, which, as expected, are both downward sloping. Apparently, they intersect only once and so, equilibrium is unique in this case. The dotted curves depict the new equilibrium when $s$ falls to 0.05 . Note that when $T$ is fixed at 1 , the equilibrium is solely determined by the $F E$ curve at $T=1$. In this case, the decline in search cost, which gives rise to the downward shift of the $F E$ curve in figure 3 , lowers the pace of new production introduction, as established in proposition 3 , from $e=11$ to $e=9$ as a result of the erosion of market power bought about by a larger $\bar{\theta}$. But the decline in search cost shall make more frequent search optimal at each $e$, as shown by the downward shift of the $C S$ curve. In this example, the new equilibrium, point $B$, is at a larger $e$ and a smaller $T$, when compared to the initial equilibrium, point $A$.

The 6 panels of figure 4 show that this tendency can be a rather common occurrence. Under the same parameterization used in the analysis in figure 3, figures $4 \mathrm{a}$ and $4 \mathrm{~b}$ respectively show that as $s$ declines, $T$ falls and $e$ rises throughout the range of 
search cost under consideration. In the meantime, figure 4c shows that, as expected, consumers respond to the decline in $s$ by raising $\bar{\theta}$, and this should weaken the market power of firms. Notwithstanding the erosion of market power, the equilibrium price, as shown in figure $4 \mathrm{~d}$, goes up. This apparent anomaly can be explained by the fact that the lowering of $T$, by virtue of shortening the shelf-life of each brand, can result in a smaller $e T$, the number of competing brands in each period of time, which serves to lower the intensity of price competition, and this is shown in figure $4 \mathrm{~d}$. In this example, this positive effect on the equilibrium price dominates the negative effect of a larger $\bar{\theta}$. Nevertheless, figure 4e shows that consumer welfare, as measured by the expected discounted utility of consumption, $\frac{\bar{\theta}-p}{1-\beta^{K-1}}$, does goes up following the decline in $s$.

To summarize the discussion, I note that:

Proposition 4 Where the optimal search frequency on the part of consumer is tied to the pace at which new products are introduced, a low search cost can result in a more rapid pace of new product introduction in equilibrium, despite the erosion of market power brought about by the low search cost. 
(a) Search freawency

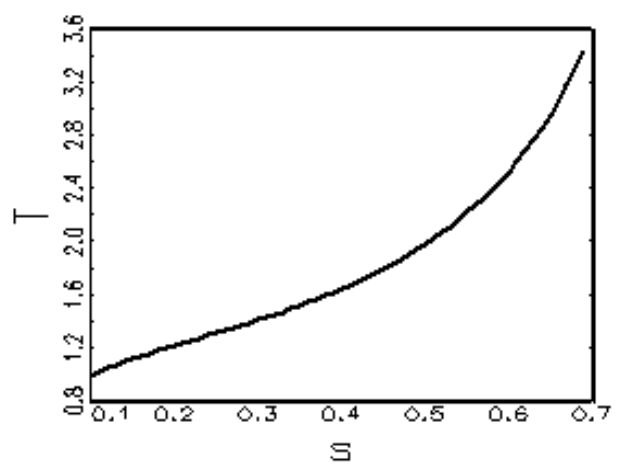

(c) Reservation match

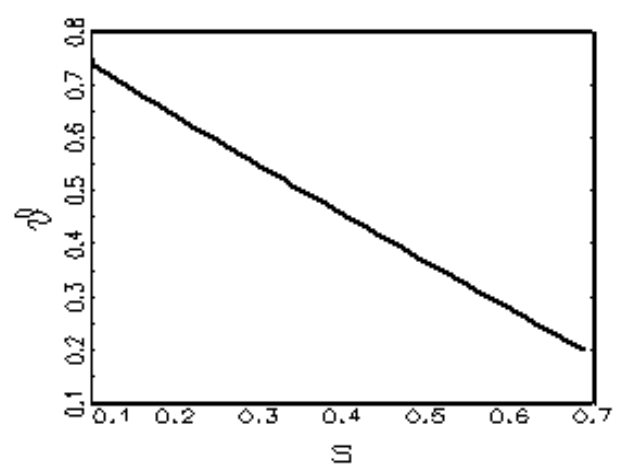

(d) Intemsity of competition

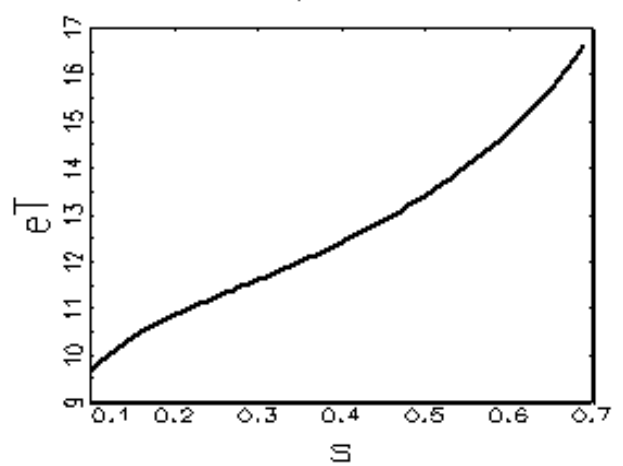

(b) Rate of entry

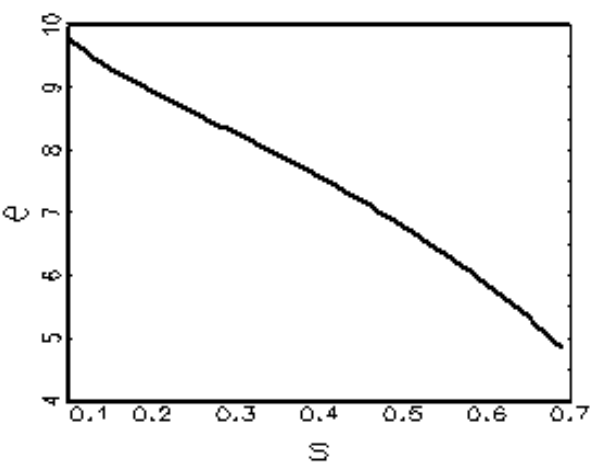

(d) Price

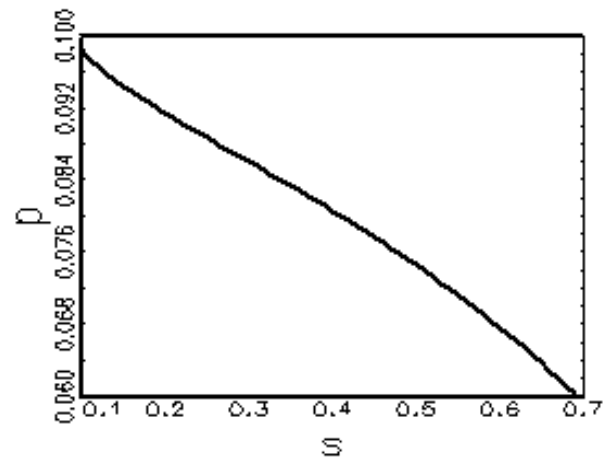

(e) Consumer welfare

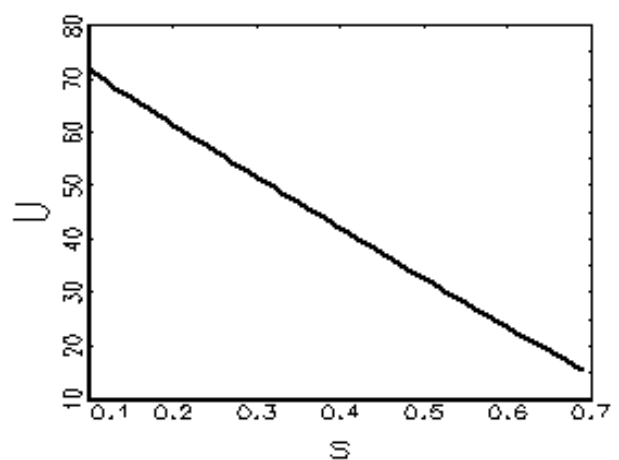

Figure 4: Comparative statics of search cost 


\section{SOCIAL OPTIMUM}

In this section, I shall examine the social planner's choice of the rate of new product introduction subject to the optimal search of consumers and the pricing rule in the monopolistic competition. Formally, the planner's objective is assumed to be

$$
\mathcal{S}=\max _{e}\left\{\frac{\bar{\theta}-p}{1-\beta^{K-1}}-\frac{c e}{1-\beta}+\frac{e(\Pi+c)}{1-\beta}\right\}
$$

subject to (6), (8), and (15). The first term is the expected discounted lifetime utility of consumption of the representative consumer, whereas the second term is the present value of the cost of having $e$ new brands introduced in each period. The difference between them is thus the net social benefit if brands are purchased at the marginal cost of production. But when the planner must respect the pricing rule in the monopolistic competition, brands are not purchased at marginal cost, and from the social point of view the profits made by firms can count toward paying for the fixed cost of product introduction to a certain extent. In view of the constraint of non-marginal cost pricing, we must add back the present value of profits (before fixed cost) to arrive at an accurate account of the net social benefit.

To proceed, denote the solutions of $\{\bar{\theta}, T, e\}$ to the system (6), (8), and (15) as $\bar{\theta}=\bar{\theta}(e), T=T(e)$, and $p=p(e)$. For a sufficiently small $\frac{1-\beta^{T}}{\left(1-\beta^{K-1}\right) \beta^{T}},{ }^{13}$

$$
\frac{d \bar{\theta}(e)}{d e}>0, \frac{d T(e)}{d e}<0, \text { and } \frac{d}{d e}[\bar{\theta}(e)-p(e)]>0 .
$$

Next, rearranging (19),

$$
\mathcal{S}=\max _{e}\left\{\frac{\bar{\theta}(e)-p(e)}{1-\beta^{K-1}}+\frac{e \prod(e)}{1-\beta}\right\} .
$$

\footnotetext{
${ }^{13}$ From proposition 1 and holding $p$ constant, the signs of the three derivtives obtain. It can be seen from (6) or (18) that the effects of an endogenous $p$ on the signs of these derivatives can be kept to minimum for a sufficiently small $\frac{1-\beta^{T}}{\left(1-\beta^{K-1}\right) \beta^{T}}$.
} 
It can immediately be ascertained that the possibly multiple equilibria can be Pareto ranked, with the low level equilibria strictly dominated by the high level equilibria. This is the case since in all equilibria, $\Pi(e)=0$ but the high level equilibria are associated with higher $\bar{\theta}(e)-p(e)$.

The next logical next is to determine whether a given equilibrium may be dominated by an off-equilibrium allocation, and relatedly whether even the highest level equilibrium can be improved upon. As in the usual model of monopolistic competition, there is no unambiguous answer to the question. First there is the imperfect appropriability of the returns to introducing a new brand, whereby a firm may only earn a fraction of the social benefits of new product introduction. And this effect tends to give rise to insufficient entry. On the other hand, there is also the wellknown business stealing effect, whereby the entry of a brand lowers the profits to be earned from all others when the intensity of competition rises, which tends to result in excessive entry. Peculiar to this model are two effects associated with how an entry of a brand may affect the profits of others through the feedback from consumer search to firms' profits. The first is about how when $e$ goes up, the increase in reservation match lowers the profits of firms. This effect has the same sign as the usual business stealing effect of monopolistic competition. On the other hand, an increase in $e$, when lowering the frequency of search, could yield greater profits for firms. This last effect makes suboptimal entry more likely in this model than in the standard model of monopolistic competition. ${ }^{14}$

\section{CONCLUDING REMARKS}

Recall that the competitive pressure hypothesis as discussed in the introductory section implies a negative correlation between the stock market value of firms and the rate of innovation. Clearly, no such relationship follows from the market turnover

\footnotetext{
${ }^{14}$ Each of these effects are the individual terms from differentiating (20) with respect to $e$.
} 
hypothesis examined in this paper. This difference provides the basis to distinguish the two hypotheses empirically. In this regard, Jovanovic and Rousseau (2001) show that historically, technological waves were indeed associated with stock market booms. While this in itself is hardly any evidence to support the market turnover hypothesis over the competitive pressure hypothesis, it does point out the difficulty facing the latter.

A possible objection to the analysis is that the advance of ICT in the new economy mainly serves to lower the marginal cost of search instead of the fixed cost that is the object of the comparative statics analysis in this paper. I shall argue that the main results of this paper remain essentially intact in an environment with both positive fixed and marginal costs of search, and where the comparative statics are concerned with the marginal cost of search. In this model, it remains optimal for consumers to initiate search periodically instead of in every period of time because of the fixed cost of initiating search. A falling marginal cost of search, in raising the expected payoff of a shopping trip, would make consumers search more frequently, giving rise to an increase in profits firms may earn from introducing new products. The qualitative results should be the same as those of a falling fixed cost of search. The consumer search problem in this model, however, would be considerably more complicated to analyze, as its solution would no longer be characterized by a stationary reservation match even when brands are charged the same price over time. Consider two consumers who have both sunk the fixed cost of search. The consumer who is nearing exhausting the available offers should be more willing to accept a given match over paying for another draw than the consumer who has many offers yet to be sampled. These complications are best to be abstracted away in an already rather complex model by assuming a zero marginal cost of search. 


\section{APPENDIX}

\section{Proof of Lemma 1}

By (3) and (5),

$$
w(\theta)=\max \left\{\theta-p+\beta^{K} U(1), \bar{\theta}-p+\beta^{K} U(1)\right\} .
$$

By (4) then,

$$
W(t)=\int^{\bar{\theta}} \bar{\theta} d F(\theta)^{e t}+\int_{\bar{\theta}} \theta d F(\theta)^{e t}-p+\beta^{K} U(1)
$$

Integrating by part yields the formula in the lemma.

\section{Proof of Lemma 2}

By lemma 1

$$
\begin{aligned}
& (W(t+1)-W(t))-(W(t)-W(t-1)) \\
= & \int_{\bar{\theta}}\left[F(\theta)^{e t}-F(\theta)^{e(t+1)}-F(\theta)^{e(t-1)}+F(\theta)^{e t}\right] d \theta \\
= & \int_{\bar{\theta}} F(\theta)^{e(t-1)}\left(1-F(\theta)^{e}\right)\left(F(\theta)^{e}-1\right) d \theta<0 .
\end{aligned}
$$

Now suppose the consumer finds initiating search optimal at some $t_{1}$, i.e.,

$$
W\left(t_{1}\right)-s \geq \beta U\left(t_{1}+1\right)
$$

but not optimal in the period afterward, i.e.,

$$
W\left(t_{1}+1\right)-s<\beta U\left(t_{1}+2\right),
$$

contrary to the assertion of the lemma. By definition,

$$
\begin{aligned}
\beta U\left(t_{1}+1\right) & =\beta \max \left\{\beta U\left(t_{1}+2\right), W\left(t_{1}+1\right)-s\right\} \\
& >\beta\left(W\left(t_{1}+1\right)-s\right)
\end{aligned}
$$


where the inequality follows from (23). Combining (22) and (24) yields

$$
W\left(t_{1}\right)-s>\beta\left(W\left(t_{1}+1\right)-s\right) .
$$

If initiating search is optimal at $t_{1}$, it must also be optimal to at some $t_{2}>t_{1}$. Otherwise, $U(t)=0$, for all $t>t_{1}$, which contradicts $U\left(t_{1}\right)=W\left(t_{1}\right)-s>0$. The lemma holds trivially if $t_{2}=t_{1}+1$. Suppose instead, $t_{2}>t_{1}+1$. Now since initiating search is assumed not optimal at $t_{1}+1$, but optimal at some $t_{2}>t_{1}+1$, there must be some $t_{3} \in\left[t_{1}+1, t_{2}\right)$, where going shopping is not optimal in the period, i.e.

$$
W\left(t_{3}\right)-s<\beta U\left(k_{3}+1\right)
$$

but becomes optimal in the period afterward, i.e.

$$
\begin{aligned}
\beta U\left(t_{3}+1\right) & =\beta \max \left\{\beta U\left(t_{3}+2\right), W\left(t_{3}+1\right)-s\right\} \\
& \leq \beta\left(W\left(t_{3}+1\right)-s\right) .
\end{aligned}
$$

Combining (26) and (27),

$$
W\left(t_{3}\right)-s \leq \beta\left(W\left(t_{3}+1\right)-s\right) .
$$

But by (21) and that $W(t)$ is increasing in $t,(25)$ and (28) cannot both hold, and so neither can both (22) and (23) hold.

The optimality of the $S-s$ type policy, as stated in part (b), is a straightforward implication of part (a).

\section{Proof of proposition 1}

At $s=0$, the left side of (8) falls below 0 at $T=1$ for each $\bar{\theta}$ lying in the interior of the support of $F(\theta)$. Hence the smallest $T \geq 1$ that satisfies $(7)$ is 1 . This proves (a). 
Next observe that the left side of (8) is increasing in $s$ without bound. Thus, at $T=1$ and for each $\bar{\theta}$ lying in the interior of the support of $F(\theta)$, one can always find a sufficiently large $s$ to make the left side of (8) to rise above zero. Hence in the optimal search, $T>1$. To check for the comparative statics, write the difference between the right and the left sides of $(6)$ as $H^{1}(T, \bar{\theta} ; s, p, e)$ and the left side of (8) as $H^{2}(T, \bar{\theta} ; s, p, e)$. In an interior solution,

$$
\begin{aligned}
& H^{1}(T, \bar{\theta} ; s, p, e)=0 \\
& H^{2}(T, \bar{\theta} ; s, p, e)=0 .
\end{aligned}
$$

Denoting partial derivatives by subscripts,

$$
\begin{aligned}
& H_{T}^{1}=0, H_{\bar{\theta}}^{1}<0, H_{s}^{1}<0, H_{p}^{1}>0, H_{e}^{1}>0 \\
& H_{T}^{2}<0, H_{\bar{\theta}}^{2}>0, H_{s}^{2}>0, H_{p}^{2}=0, H_{e}^{2}<0 .
\end{aligned}
$$

for $\beta \in(0,1)$. Note that $H_{T}^{1}=H^{2}$, and hence $H_{T}^{1}$ must vanish at the optimum. The rest of the above can all be established by simple differentiation. By the implicit function rule,

$$
\begin{array}{ll}
\frac{\partial T}{\partial s}=\frac{H_{\bar{\theta}} H_{s}^{2}-H_{\bar{\theta}}^{2} H_{s}^{1}}{J}, & \frac{\partial \bar{\theta}}{\partial s}=\frac{H_{s}^{1} H_{T}^{2}-H_{s}^{2} H_{T}^{1}}{J}<0 \\
\frac{\partial T}{\partial p}=\frac{H_{\bar{\theta}} H_{p}^{2}-H_{\bar{\theta}}^{2} H_{p}^{1}}{J}>0, & \frac{\partial \bar{\theta}}{\partial p}=\frac{H_{p}^{1} H_{T}^{2}-H_{p}^{2} H_{T}^{1}}{J}>0 \\
\frac{\partial T}{\partial e}=\frac{H_{\bar{\theta}} H_{e}^{2}-H_{\bar{\theta}}^{2} H_{e}^{1}}{J}, & \frac{\partial \bar{\theta}}{\partial e}=\frac{H_{e}^{1} H_{T}^{2}-H_{e}^{2} H_{T}^{1}}{J}>0
\end{array}
$$

where $J=H_{T}^{1} H_{\bar{\theta}}^{2}-H_{\bar{\theta}}^{1} H_{T}^{2}<0$. It turns out that the inequalities in (29) suffice to sign all of the above except for $\frac{\partial T}{\partial s}$ and $\frac{\partial T}{\partial e}$. To proceed, combine (6) and (8) to obtain

$$
\frac{\bar{\theta}-p}{1-\beta^{K-1}} \frac{\ln \beta}{\beta^{T}}-e \int_{\bar{\theta}} F(\theta)^{e T} \ln F(\theta) d \theta=0,
$$

whose left side is decreasing in $\bar{\theta}$ and in $T$. With $s$ not entering the equation, this implies that variations in $s$ would either (a) increase $T$ and decrease $\bar{\theta}$ or (b) decrease 
$T$ and increase $\bar{\theta}$. Since $\frac{\partial \bar{\theta}}{\partial s}<0$, we have $\frac{\partial T}{\partial s}>0$. As to $\frac{\partial T}{\partial e}$, with $J<0$, the sign is the same as the sign of $\Phi=H_{\bar{\theta}}^{2} H_{e}^{1}-H_{e}^{2} H_{\bar{\theta}}^{1}$. Evaluating the partial derivatives using (6) and (8) :

$$
\begin{aligned}
\Phi= & {\left[-\frac{\left(F(\bar{\theta})^{e T}-1\right) \ln \beta}{1-\beta^{T}}-e F(\bar{\theta})^{e T} \ln F(\bar{\theta})\right] T \beta^{T} \int_{\bar{\theta}} F(\theta) \ln F(\theta) d \theta-\left(\beta^{T} F(\bar{\theta})^{e T}\right.} \\
& \left.-\frac{1-\beta^{T+K-1}}{1-\beta^{K}}\right)\left[\left(\frac{-T \ln \beta}{1-\beta^{T}}-1\right) \int_{\bar{\theta}} F(\theta) \ln F(\theta) d \theta-e T \int_{\bar{\theta}} F(\theta)^{e T}(\ln F(\theta))^{2}\right] \\
< & {\left[-\frac{\left(F(\bar{\theta})^{e T}-1\right) \ln \beta}{1-\beta^{T}}-e F(\bar{\theta})^{e T} \ln F(\bar{\theta})\right] T \beta^{T} \int_{\bar{\theta}} F(\theta) \ln F(\theta) d \theta-} \\
& \left(\beta^{T} F(\bar{\theta})^{e T}-1\right)\left[\left(\frac{-T \ln \beta}{1-\beta^{T}}-1\right) \int_{\bar{\theta}} F(\theta) \ln F(\theta) d \theta-e T \int_{\bar{\theta}} F(\theta)^{e T}(\ln F(\theta))^{2}\right] \\
= & {\left[-T \ln \beta-e T \beta^{T} F(\bar{\theta})^{e T} \ln F(\bar{\theta})-1+\beta^{T} F(\bar{\theta})^{e T}\right] \int_{\bar{\theta}} F(\theta) \ln F(\theta) d \theta } \\
& -e T\left(1-\beta^{T} F(\bar{\theta})^{e T}\right) \int_{\bar{\theta}} F(\theta)^{e T}(\ln F(\theta))^{2} .
\end{aligned}
$$

The second line of the final expression is obviously negative for all $\beta \in(0,1)$. The first line tends to negative infinity as $\beta \rightarrow 0$ and is increasing for all $\beta$. Hence $\Phi$ is guaranteed to be negative for all $\beta \in(0,1)$ if it is non-positive at $\beta=1$; i.e. if

$$
\left[-e T F(\bar{\theta})^{e T} \ln F(\bar{\theta})-1+\beta^{T} F(\bar{\theta})^{e T}\right] \int_{\bar{\theta}} F(\theta) \ln F(\theta) d \theta \leq 0 .
$$

Now from (6), $\bar{\theta}$ approaches the upper bound of $B$ as $\beta \rightarrow 1$, and the above vanishes. This establishes the negativity of $\frac{\partial T}{\partial e}$.

\section{Proof of lemma 3}

Differentiate (15) to obtain:

$$
\frac{\partial p}{\partial \bar{\theta}}=\frac{-F(\bar{\theta})^{e T-1}\left(f(\bar{\theta})+p f^{\prime}(\bar{\theta})\right)}{f(\bar{\theta}) F(\bar{\theta})^{e T-1}+\int_{\bar{\theta}} f(\theta) d F(\theta)^{e T-1}} .
$$


If $f^{\prime}(\bar{\theta}) \geq 0, \frac{\partial p}{\partial \bar{\theta}}<0$ for sure. Suppose $f^{\prime}(\bar{\theta})<0$. Since $p \leq \bar{\theta}$, for otherwise, $\Pi<0$, we have

$$
\frac{\partial p}{\partial \bar{\theta}} \leq \frac{-F(\bar{\theta})^{e T-1}\left(f(\bar{\theta})+\bar{\theta} f^{\prime}(\bar{\theta})\right)}{f(\bar{\theta}) F(\bar{\theta})^{e T-1}+\int_{\bar{\theta}} f(\theta) d F(\theta)^{e T-1}}
$$

the negativity of which is guaranteed by the condition stated in the lemma. Alternatively, one may use the first order condition of (14) to derive (31). If particular, denote this first order condition as

$$
\Psi\left(p, p_{i}, \bar{\theta}, e T\right)=0
$$

By the implicit function rule,

$$
\frac{\partial p_{i}}{\partial \bar{\theta}}=\frac{\partial \Psi\left(p, p_{i}, \bar{\theta}, e T\right) / \partial \bar{\theta}}{-\partial \Psi\left(p, p_{i}, \bar{\theta}, e T\right) / \partial p_{i}}
$$

Evaluating the numerator at $p_{i}=p$,

$$
\frac{\partial p_{i}}{\partial \bar{\theta}}=\frac{-F(\bar{\theta})^{e T-1}\left(f(\bar{\theta})+p f^{\prime}(\bar{\theta})\right)}{-\partial \Psi\left(p, p_{i}, \bar{\theta}, e T\right) / \partial p_{i}} .
$$

A comparison of (31) and (33) reveals that

$$
\left.\frac{\partial \Psi\left(p, p_{i}, \bar{\theta}, e T\right)}{\partial p_{i}}\right|_{p_{i}=p}=-f(\bar{\theta}) F(\bar{\theta})^{e T-1}-\int_{\bar{\theta}} f(\theta) d F(\theta)^{e T-1}<0
$$

verifying that the second order condition for a local optimum is satisfied at $p=p_{i}$.

Let $y=e T$. Differentiate (15) with respect to $y$ to obtain:

$$
\begin{aligned}
\frac{\partial p}{\partial y}= & \left(y f(\bar{\theta}) F(\bar{\theta})^{y-1}+y \int_{\bar{\theta}} f(\theta) d F(\theta)^{y-1}\right)^{-1} \times \\
& \left\{\left(1-F(\bar{\theta})^{y}+y F(\bar{\theta})^{y} \ln F(\bar{\theta})\right)\left(-f(\bar{\theta}) F(\bar{\theta})^{y-1}-\int_{\bar{\theta}} f(\theta) d F(\theta)^{y-1}\right)\right. \\
& \left.-\int_{\bar{\theta}} f(\theta)^{2} F(\theta)^{y-2}(1+(y-1) \ln F(\theta)) d \theta\right\} .
\end{aligned}
$$

Since $1-F(\bar{\theta})^{y}+y F(\bar{\theta})^{y} \ln F(\bar{\theta})>0$ for all $\bar{\theta}$ in the interior of the support of $F()$, $\frac{\partial p}{\partial y}<0$ for sure if $\int_{\bar{\theta}} f(\theta)^{2} F(\theta)^{y-2}(1+(y-1) \ln F(\theta)) d \theta>0$ that is the condition of the lemma. 


\section{Proof of lemma 4}

Set $p_{i}=p$ in (14), substitute (15) into the resulting expression, and differentiate with respect to $\bar{\theta}$ to obtain

$$
\begin{aligned}
\frac{\partial \Pi}{\partial \bar{\theta}}= & \frac{\Pi F(\bar{\theta})^{e T}}{T+(K-1)\left(1-F(\bar{\theta})^{e T}\right)} \frac{e T}{1-F(\bar{\theta})^{e T}}\left\{\left(-2 f(\bar{\theta})-p f^{\prime}(\bar{\theta})\right) \times\right. \\
& \left.\left(T+(K-1)\left(1-F(\bar{\theta})^{e T}\right)\right)+(K-1) f(\bar{\theta})\left(1-F(\bar{\theta})^{e T}\right)\right\},
\end{aligned}
$$

which is negative for sure if $f^{\prime}(\bar{\theta}) \geq 0$. If $f^{\prime}(\bar{\theta})<0$, we may use the condition in lemma 3a to establish the negativity of the above.

Use (16) to write $\Pi$ as

$$
\Pi=\frac{1-\beta^{T}}{1-\beta} \frac{p}{T}\left(\frac{e T}{1-F(\bar{\theta})^{e T}}+e(K-1)\right)^{-1}-c,
$$

which is decreasing in $e$, holding $p$ constant. Next rewrite $\Pi$ as

$$
\Pi=\frac{p}{(1-\beta) e}\left(\frac{T}{1-\beta^{T}}\left(\frac{T}{1-F(\bar{\theta})^{e T}}+K-1\right)\right)^{-1}-c
$$

which is decreasing in $T$, holding $p$ constant. That $p$ is similarly decreasing in $e$ and $T$ confirms that increases in $e$ and $T$ result in a lower $\Pi$. 


\section{REFERENCES}

[1] Anderson, Simon P. and Regis Renault, 1999, "Pricing, Product Diversity, and Search Costs: A Bertrand- Chamberlin-Diamond Model," Rand Journal of Economics 30, 719-35.

[2] Arrow, Kenneth, 1962, "Economic Welfare and the Allocation of Resources for Invention," in R. Nelson (ed.), The Rate and Direction of Inventive Activity, NBER, Princeton University Press.

[3] Bulow, Jeremy, 1982, "Durable goods monopolies," Journal of Political Economy 90, $314-332$.

[4] Diamond, Peter A., 1971, "A model of price adjustment", Journal of Economic Theory $3,156-168$.

[5] — 1982, "Aggregate demand management in search equilibrium," Journal of Political Economy 90, 881-894.

[6] Fishman, Arthur and Rafael Rob, 1995, "The Durability of Information, Market Efficiency and the Size of firms," International Economic Review 36, 19-36.

[7] Jovanovic, Boyan and Peter Rousseau, 2001, "Vintage organization capital," NBER working paper 8166 .

[8] Kohn, Meir G. and Steven Shavell, 1974, "The Theory of Search," Journal of Economic Theory 9, 93-123.

[9] Lippman, Steven A. and J.J. McCall, 1976, "The Economics of Job Search: A Survey," Economic Inquiry 14, 347-368.

[10] Pereira, Pedro, 2001, "Market power, cost reduction and consumer search," International Journal of Industrial Organization 19, 1457-1473. 
[11] Reinganum, Jennifer F., 1979, "A simple model of price dispersion," Journal of Political Economy 87, 851-858.

[12] Tse, Chung Yi, 2003, "Search frictions, market power, and long run growth," Journal of Economic Theory, in press.

[13] Wolinsky, Asher, 1984, "Product differentiation with imperfect information," Review of Economic Studies 51, 53-61. 